\title{
Multi-Objective Controller Design for a Space Launcher
}

\author{
M. Abbas-Turki* \\ G. $\operatorname{Duc}^{\dagger}$ \\ ENS de Cachan, F94235 Cachan, France \\ Supélec, F91192 Gif-sur-Yvette, France \\ B. Clement $\ddagger$ \\ CNES, F91023 Evry, France
}

The design of an autopilot for a space launcher during the atmospheric stage is known to be a hard control problem, which contains an important number of conflicting specifications. This difficulty is increased by the presence of flexible modes, some of them acting inside the bandwidth, and parameter uncertainties.

To solve such a problem, a multi-objective approach is proposed in this paper, which combines the Youla parameterization, suitable translations of the different specifications into linear matrix inequalities (LMI), and convex optimisation using a cutting plane algorithm (CPA).

These tools together can handle high order controllers without numerical difficulties. They can also efficiently provide an answer to the crucial question of the feasibility or unfeasibility of the specifications. Although they can be used for a wide class of multi-objective problems, the paper doesn't particularly focus on the theoretical developments (which can be found in recently published works) but rather on their application to the space launcher control problem.

\section{Nomenclature}

G Centre of gravity

GXYZ Guidance attitude reference

*Assistant Professor, SATIE 61 Avenue du Président Wilson; m.abbas-turki@orange.fr

†Professor, Automatic Control Department, 3 rue Joliot-Curie; Gilles.Duc@supelec.fr

${ }^{\ddagger}$ Research Engineer, Direction des Lanceurs, Rond Point de l'Espace; benoit.clement@cnes.fr 
Gxyz Launcher reference

$P_{c} \quad$ Useful thrust for piloting

$\beta \quad$ Thruster angle of deflection

$\psi \quad$ Deviation angle around axis w.r.t the guidance attitude reference

W Wind velocity

$\dot{z} \quad$ Lateral drift rate

$i \quad$ Angle of attack

$V \quad$ Absolute velocity

$V_{R} \quad$ Relative velocity

$\eta_{i} \quad$ Generalized coordinate of the i-th bending mode

$\omega_{i}, \xi_{i} \quad$ Natural frequency and damping of the i-th bending mode

$\omega_{T}, \xi_{T} \quad$ Natural frequency and damping of the actuator

$\omega_{G}, \xi_{G} \quad$ Natural frequency and damping of the gyrometer

$h_{t}(i) \quad$ Deviation of the i-th bending mode in front of the thruster

$h_{c l}^{\prime}(i) \quad$ Slope of the modal deformation in front of the inertial measurement unit

$h_{G y}^{\prime}(i) \quad$ Slope of the modal deformation in front of the gyrometer

\section{Introduction}

ORE attention has been paid in recent years to the space launcher controller design M problem (see for instance ${ }^{1,2,3,4,5,6}$ ), where different modern synthesis methods are considered starting from LQG synthesis to multi-objectives design. This interest is motivated by the difficulty to control the space launcher during the atmospheric stage, where each launching is becoming a prowess. This difficulty is explained by the economics constraints, since the space launcher is a disposable machine so that the strategy is to reduce the expenses on the structure and also to make it lighter in order to launch heavier satellites. All these arrangements lead to a difficult control problem and perhaps even to an unfeasible one.

The multi-objectives synthesis is becoming an unavoidable technique of control for space launcher problems, because all other methods add some conservatism (as $\mathcal{H}_{\infty^{-}}$synthesis) or yield to non robust controllers (as $\mathcal{H}_{2}$-synthesis). The combination of different criteria enables to precisely specify the constraints imposed by manufacturer, but to this end they have to be translated using a similar and numerically tractable formalism, which is still an important area of research. ${ }^{7,8,9,10}$ The worrying point is that in major cases the criteria are unfortunately conflicting. Therefore the main goal of the multi-objectives design is to express the criteria in such way that the conservatism introduced by translating the manufacturer 
specifications is as less as possible, in order to reduce the conflict between them and to lead to a satisfying controller.

For this goal the methodology used to translate the constraints must be able to propose a mathematical formulation which is as close as possible to the manufacturer specifications. This part of the synthesis procedure appears undeniably crucial for a hard control problem, such as the one of the space launcher.

The translation of the constraints is also depending on the controller synthesis tools. Consequently the choice of a synthesis method should allow to consider a set of controllers as large as possible. For linear plants, the Youla parameterization ${ }^{11,7}$ defines a convex set describing all stabilizing controllers, so that no possible solution is lost.

To preserve the convexity property brought by the Youla parameterization, the manufacturer specifications can be translated using the Linear Matrix Inequalities (LMI) framework. ${ }^{12}$ However, if LMI formulations are well known for $\mathcal{H}_{2}$ and $\mathcal{H}_{\infty}$ norm constraints or for pole placement for instance, ${ }^{8}$ such constraints are only indirect translations of the specifications in most cases (for instance, pole placement handles imperfectly a requirement on the settling time). In this paper, recently proposed formulations ${ }^{13}$ are used for time-domain templates, stability margins and constraints in a given frequency range, together with $\mathcal{H}_{2^{-}}$ norm constraints.

The application of the Youla parameterization induces a huge controller order, especially for harsh control problems. Moreover, most commonly used LMI formulations generally require introducing matrices of the same order as the closed-loop plant, so that the problem can become numerically infeasible. In order to avoid such additional variables, Kao ${ }^{14}$ presents a method based on the eigenvalues of some Hamiltonian matrix, and the application of a Cutting Plane Algorithm (CPA) instead of using the interior point algorithm. Although this method is more sensitive to numerical conditioning, it is less affected by the order of the plant.

In this paper the problem of using the CPA is not detailed (more information can be found in Ref. 13), but a brief presentation of the algorithm is given, aiming to understand the different tools and results given in the paper. The paper is devoted to surround the control problem of a space launcher during the atmospheric stage, using new LMI formulations and a suitable analysis to decide the feasibility or unfeasibility of the specifications.

The paper is organized as follows: section 2 presents the problem formulation with the 
model of the space launcher during the atmospheric stage and the different objectives stipulated by the manufacturer; section 3 contains a brief presentation of the Youla parametrization and the CPA ; section 4 explains the translation of the control objectives into LMI constraints and deals with intrinsic properties of the control problem of the launcher ; in section 5 the numerical results are discussed. The last section is the conclusion.

\section{Problem formulation}

\section{A. Aerospace launcher modelling}

The dynamics of the Europeean space launcher are described by the variables given on figure 1 and the nomenclature. Only the yaw axis is considered, assuming that the other axes (roll and pitch axes) are decoupled. The sloshing effect are also neglected. ${ }^{4}$

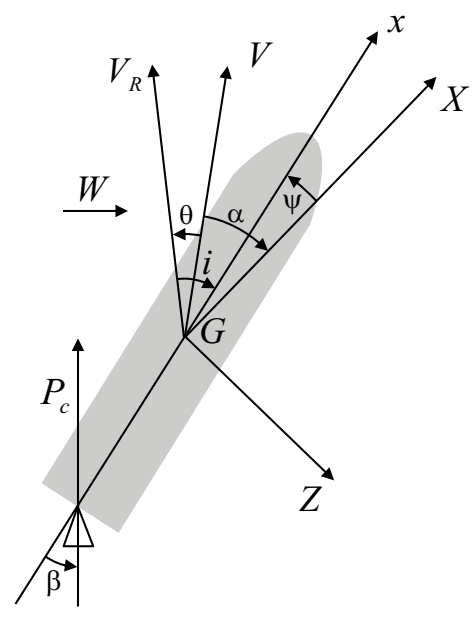

Figure 1. Two dimension launcher representation

The model of the launcher is derived according to the block-diagram given on figure $2 .^{\mathrm{a}}$ It is decomposed into four parts:

Rigid launcher model:

Assuming all angles remain small, the linearised dynamics of the launcher can be de-

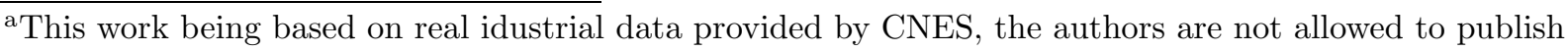
the numerical values of the parameters and the specifications; all numbers have been therefore removed from the figures but the later will appear explicitely. 


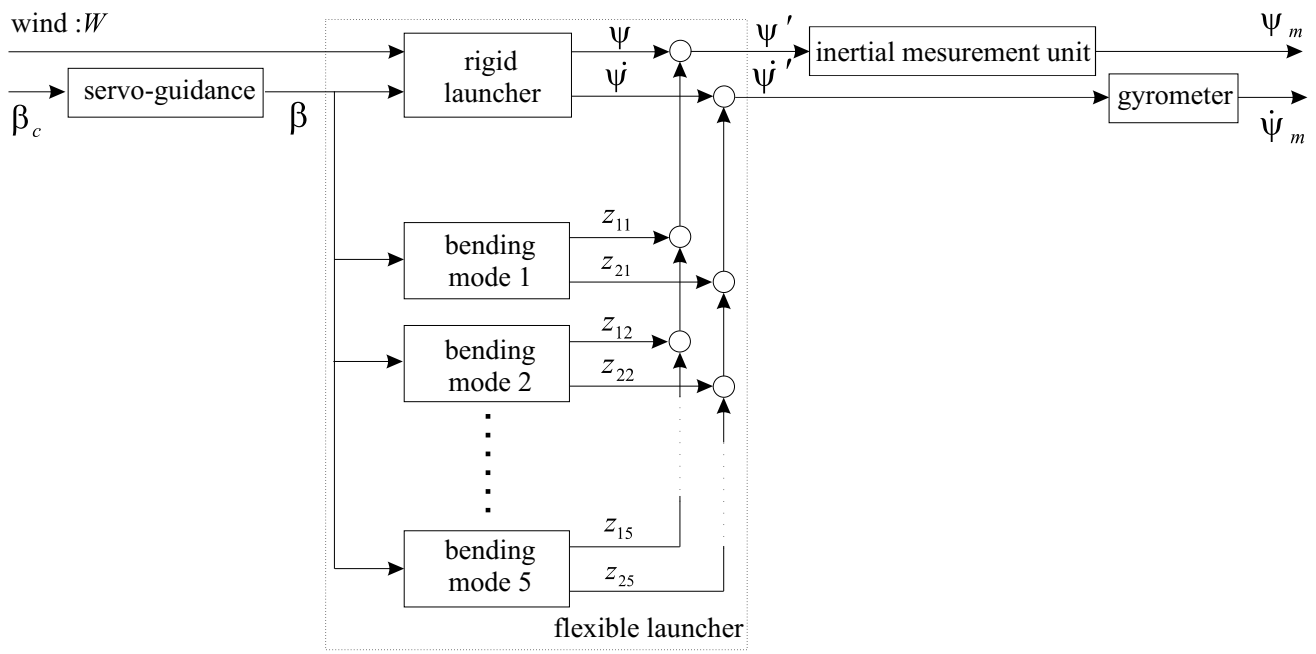

Figure 2. Block diagram of the launcher model

scribed by the following state-space model:

$$
\frac{d}{d t}\left(\begin{array}{c}
\psi \\
\dot{\psi} \\
\dot{z}
\end{array}\right)=\left(\begin{array}{ccc}
0 & 1 & 0 \\
A_{6} & 0 & \frac{A_{6}}{V} \\
a_{1} & 0 & a_{2}
\end{array}\right)\left(\begin{array}{c}
\psi \\
\dot{\psi} \\
\dot{z}
\end{array}\right)+\left(\begin{array}{cc}
0 & 0 \\
K_{1} & -\frac{A_{6}}{V} \\
a_{3} & -a_{2}
\end{array}\right)\left(\begin{array}{c}
\beta \\
W
\end{array}\right)
$$

where $\psi$ is the deviation angle and $\dot{z}$ the lateral drift rate; $\beta$ is the thruster angle of deflection and $W$ the wind velocity. Parameters $A_{6}$ and $K_{1}$, which are slowly varying, are respectively the coefficients of aerodynamic efficiency and thruster efficiency. The control law being designed using a LTI model, most modeling uncertainties can be handled by considering uncertainties on these two parameters.

Moreover, the angle of attack $i$ is defined by:

$$
i=\psi+\frac{\dot{z}-W}{V}
$$

Flexible modes:

Each bending mode can be represented by a second-order model with natural frequency $\omega_{i}$ and damping $\xi_{i}$ :

$$
\frac{d}{d t}\left(\begin{array}{c}
\eta_{i} \\
\dot{\eta}_{i}
\end{array}\right)=\left(\begin{array}{cc}
0 & 1 \\
-\omega_{i}^{2} & -2 \xi_{i} \omega_{i}
\end{array}\right)\left(\begin{array}{c}
\eta_{i} \\
\dot{\eta}_{i}
\end{array}\right)+\left(\begin{array}{c}
0 \\
-\omega_{i}^{2} P_{c} h_{t}(i)
\end{array}\right) \beta
$$

Taking into account the first five bending modes and assuming the other ones are neg- 
ligible, the effect of the bending modes can be handled by adding pertubation terms on $\psi$ and $\dot{\psi}$ :

$$
\begin{aligned}
\psi^{\prime} & =\psi+\sum_{i=1}^{5} h_{c l}^{\prime}(i) \eta_{i} \\
\dot{\psi}^{\prime} & =\dot{\psi}+\sum_{i=1}^{5} h_{G y}^{\prime}(i) \dot{\eta}_{i}
\end{aligned}
$$

Actuator:

The servo-guidance is modelled as a second order system:

$$
\ddot{\beta}+2 \xi_{T} \omega_{T} \dot{\beta}+\omega_{T}^{2} \beta=\omega_{T} \beta_{c}
$$

where $\xi_{T}, \omega_{T}$ and $\beta_{c}$ are respectively the damping, the natural frequency and the commanded angle of deflection.

\section{Sensors:}

Two measures are available: the deviation angle $\psi$ is measured using an inertial measurement unit, which is considered as a constant gain; its derivative $\dot{\psi}$ is obtained using a gyrometer which is modelled as a second-order system:

$$
\psi_{m}^{(3)}+2 \xi_{G} \omega_{G} \ddot{\psi_{m}}+\omega_{G}^{2} \dot{\psi_{m}}=\omega_{G} \dot{\psi}^{\prime}
$$

where $\xi_{G}, \omega_{G}$ and $\dot{\psi_{m}}$ are respectively the damping, the natural frequency and the measure obtained at the output of the sensor.

\section{B. Design specifications}

As explained above, the main modeling uncertainties on the rigid dynamics are captured by considering uncertainties on parameters $A_{6}$ and $K_{1}$; all parameters defining the bending modes are also uncertain. Some worst case configurations, where the combination of parameter values is particularly critical, have been identified. ${ }^{5}$ They are listed in Table 1 and are used to evaluate the performance and robustness of the control law.

The control problem ${ }^{15}$ is to design a discrete-time controller (with fixed sampling period $T$ ) according to the following objectives:

- the angle of attack $i$ has to be limited to some specified value $i_{\text {max }}$ despite measurement noise and wind and gust disturbances (typical wind profiles are shown on figure 3),

- closed-loop stability is obtained with minimal decreasing and increasing gain margins $D G M$ and $I G M$ and a delay margin at least equal to the sample time $T$, 
Table 1. Worst cases configurations.

\begin{tabular}{|c|c|c|c|c|}
\cline { 2 - 5 } \multicolumn{1}{c|}{} & $K_{1}$ & $A_{6}$ & modal deformation & $\omega_{i}$ \\
\hline parametric & $\pm 10 \%$ & $\pm 40 \%$ & $\begin{array}{c} \pm 20 \% \\
( \pm 30 \% \text { for the } \\
\text { first mode })\end{array}$ & $\begin{array}{c} \pm 10 \% \\
( \pm 15 \% \text { for the } \\
\text { first mode })\end{array}$ \\
\hline 1 & + & - & + & + \\
\hline 2 & + & - & + & nominal \\
\hline 3 & + & - & + & - \\
\hline 4 & - & + & + & + \\
\hline 5 & - & + & + & nominal \\
\hline 6 & - & + & + & - \\
\hline
\end{tabular}

- a minimal open-loop attenuation $X_{H F} \mathrm{~dB}$ (with $X_{H F}<0$ ) is obtained for all bending modes,

- the angle of attack, the angle of deflection and its velocity must remain below some specified values, $i_{\max }, \beta_{\max }$ and $\dot{\beta}_{\max }$ respectively,

- in order to limit the consumption, the following indicator has to remain below some specified value $C_{\max }$ :

$$
C=\sum_{k=T_{\text {init }}}^{T_{f i n}}\left|\beta_{k+1}-\beta_{k}\right|
$$

where $T_{\text {init }}$ and $T_{\text {fin }}$ are the initial and final time of the flight,

these specifications being satisfied for the nominal plant and for all worst case configurations listed in Table 1.

Remark 1 In the initial problem (solved for instance in Refs. 5, 4, 16), the attenuation constraint on the bending modes was relaxed for the first one. Following these results, it has been added here both to examine its feasibility together with the other constraints and to evaluate the efficiency of the proposed approach.

\section{Synthesis model}

A particular model has to be chosen to perform the controller design. The actuator and sensor dynamics being faster than those of the launcher, their dynamics will not be taken into account in the synthesis model; regarding the high complexity of the complete model and the design specifications together with the wide range of uncertainties, only the rigid 


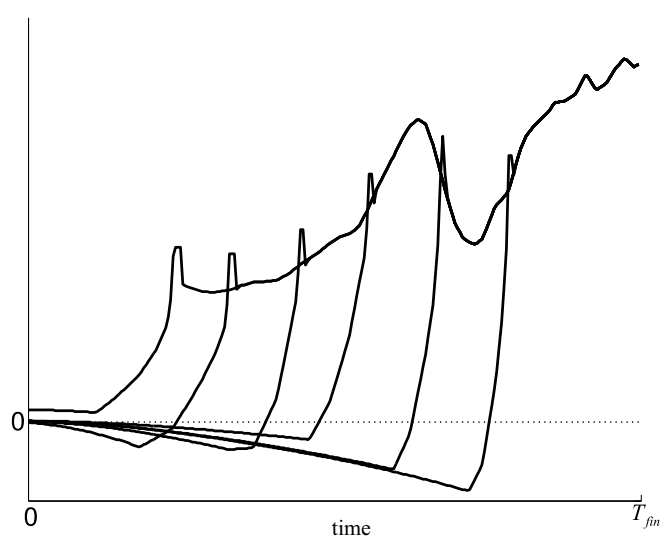

Figure 3. Wind profiles

model (1) without bending modes will be used, with nominal values for $A_{6}$ and $K_{1}$. Since a discrete-time controller is looked for, this model is discretized with sampling period $T$ by considering a zero-order hold on the control input $\beta$.

Of course the design specifications will be checked by considering the complete model (with five bending modes together with the actuator and sensor dynamics), for all configurations listed in Table 1.

\section{The Youla parameterization and the Cutting Plane Algorithm}

\section{A. Youla parameterization}

Since the work of Ref. 17, the Youla parameterization has often been used in multiobjective control problems. ${ }^{18,19}$ Consider a continuous or discrete-time plant $G$ with state space realization:

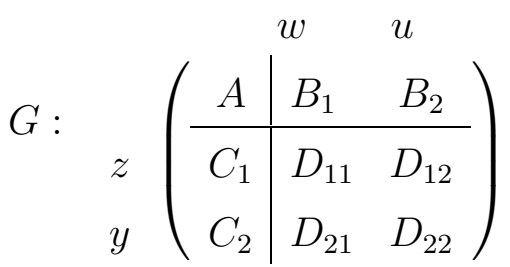

where $z$ is the output to be controlled despite disturbance $w$, using control input $u$ and measurement $y$. All stabilizing controllers are described by the Redheffer product $K=J * Q$ (see the interconnection structure of Figure 4), where the Youla parameter $Q$ is any stable transfer function. System $J$ depends both on $G_{22}$ (the transfer between $u$ and $y$ ) and an initial compensator $K_{0}$, more precisely on stable coprime factorisations $G_{22}=N M^{-1}=\tilde{M}^{-1} \tilde{N}$ and 
$K_{0}=U_{0} V_{0}^{-1}$

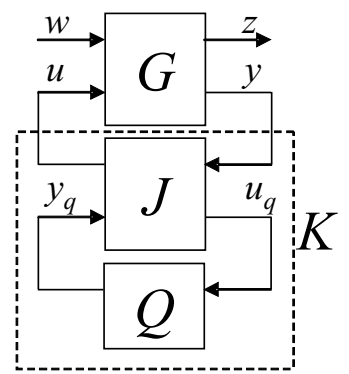

Figure 4. Closed-loop structure using Youla parameterization

An initial compensator must therefore be known to use the Youla parameterization. It can be for instance a static one or any compensator of the same order as the plant put on the LQG structure. ${ }^{20}$

The interconnection $G * J$ exhibits a transfer function identically equal to 0 between $u_{q}$ and $y_{q}$ (Figure 4 ). As an interesting result, the closed loop transfer matrix $G_{z w}$ (between input $w$ and output $z$ ) is affine in $Q$ :

$$
G_{z w}=\left(G_{11}+G_{12} U_{0} \tilde{M} G_{21}\right)+\left(G_{12} M\right) Q\left(\tilde{M} G_{21}\right):=H_{11}+H_{12} Q H_{21}
$$

In all the literature concerning the Youla parameterization and convex optimization problems, it is a usual way to approximate the Youla parameter by truncating its projection on a given basis. ${ }^{18,19}$ For MIMO models, such an approximation can be written:

$$
Q(\varsigma)=\sum_{j=1}^{m_{2}} \sum_{u=1}^{p_{2}}\left(\sum_{k=0}^{n_{q}} q_{k, j, u} Q_{k, j, u}(\varsigma)\right) e_{j} e_{u}^{T}=\sum_{m_{2}, p_{2}} Q_{j, u}(\varsigma) e_{j} e_{u}^{T}
$$

where $e_{i}$ is the unitary vector whose $\mathrm{i}$-th element is equal to $1, Q_{j, u}$ is a SISO transfer function and $\varsigma$ is either the discrete-time or Laplace operator; $\left\{Q_{k, j, u}\right\}$ is a chosen basis of stable transfer functions and $q_{k, j, u}$ are the design parameters. Let $\left(A_{Q_{j, u}}, B_{Q_{j, u}}, C_{Q_{j, u}}, D_{Q_{j, u}}\right)$ be a state-space realization of $Q_{j, u}$ : matrices $A_{Q_{j, u}}$ and $B_{Q_{j, u}}$ are fixed by the choice of $\left\{Q_{k, j, u}\right\}$, so that all the design parameters $q_{k, j, u}$ enter in $C_{Q_{j, u}}$ and $D_{Q_{j, u}}$ only.

As it can be noticed, the order of the Youla parameter rises significantly for systems with large numbers of inputs and outputs. Furthermore the state-space representation of the closed-loop plant which will be derived below is a non minimal one. For these reasons, the synthesis method must be little sensitive to the state-space order.

The design variables must appear only in the output matrices $C_{z w}$ and $D_{z w}$ of the statespace realization of the closed-loop plant $G_{z w}$, for guaranteing in most cases the linearity of the matrix inequalities constraints with respect to the design parameters. A suitable technique has been proposed by Hindi, ${ }^{18}$ which consists in increasing the representation of 
$G_{z w}$ using the Kronecker product. Taking (10) on $G_{z w}$ given by (9) leads to:

$$
\begin{aligned}
G_{z w} & =H_{11}+H_{12}\left(\sum_{m_{2}, p_{2}} Q_{j, u} e_{j} e_{u}^{T}\right) H_{21} \\
& =H_{11}+\sum_{m_{2}, p_{2}} Q_{j, u} \otimes T_{j, u}
\end{aligned}
$$

with: $T_{j, u}=\left(H_{12} e_{j}\right)\left(e_{u}^{T} H_{21}\right)$.

Let $\left(A_{11}, B_{11}, C_{11}, D_{11}\right)$ and $\left(A_{T_{j, u}}, B_{T_{j, u}}, C_{T_{j, u}}, D_{T_{j, u}}\right)$ be state-space representations of $H_{11}$ and $T_{j, u}$ for each values of $j, u$ respectively. The Kronecker product $Q_{j, u} \otimes T_{j, u}$ has the following state-space realization:

$$
\left(\begin{array}{c|c}
A_{Q_{j, u} \otimes T_{j, u}} & B_{Q_{j, u} \otimes T_{j, u}} \\
\hline C_{Q_{j, u} \otimes T_{j, u}} & D_{Q_{j, u} \otimes T_{j, u}}
\end{array}\right)=\left(\begin{array}{cc|c}
A_{Q_{j, u}} \otimes I_{m_{1}} & B_{Q_{j, u}} \otimes C_{T_{j, u}} & B_{Q_{j, u}} \otimes D_{T_{j, u}} \\
0 & A_{T_{j, u}} & B_{T_{j, u}} \\
\hline C_{Q_{j, u}} \otimes I_{m_{1}} & D_{Q_{j, u}} \otimes C_{T_{j, u}} & D_{Q_{j, u}} \otimes D_{T_{j, u}}
\end{array}\right)
$$

As it can be noticed from (12), all design variables enter only in matrices $C_{Q_{j, u} \otimes T_{j, u}}$, $D_{Q_{j, u} \otimes T_{j, u}}$ and consequently, from (11), they appear only in matrices $C_{z w}$ and $D_{z w}$.

This representation leads to a state space realization $\left(A_{z w}, B_{z w}, C_{z w}, D_{z w}\right)$ of $G_{z w}$ having a high order (that is $n+2 n m p_{2}+2 m p_{1} n_{Q} m_{1}$, where $n$ and $n_{Q}$ are respectively the dimensions of matrices $A, A_{Q}$ ). This means that for avoiding numerical infeasibility, all methods based on introducing a matrix having the same order as $A_{z w}$ should be avoided.

Remark 2 Scherer ${ }^{19}$ presented another technique, where a minimal form of $G_{z w}$ is used but suitable transformations are required to restore the linearity of the constraints. Note also that the transformation is done for $\mathcal{H}_{\infty}$ and $\mathcal{H}_{2}$ norm constraints, but does not apply for some of the constraints that are considered below.

\section{B. The Cutting Plane Algorithm}

This section presents a variant of the Cutting Plane Algorithm (CPA) introduced by Kao. ${ }^{14}$ Only the case of a feasibility problem is presented.

The presentation of the method is divided into two parts: the first one gives the general principle of the algorithm. The second one brings the details on the operations happening at each step. 


\section{Algorithm}

Consider the following convex feasibility problem:

$$
\text { Find } x \quad \text { subj to } \mathcal{S}_{x}>0
$$

where $x$ is the vector of decision variables, and $\mathcal{S}_{x}$ is a real symmetric matrix, affine on $x$, which expresses a set of constraints on matrix form. The problem (13) can be reformulated into an equivalent eigenvalue maximization problem:

$$
\sup _{x, y} y \quad \operatorname{subj} \text { to } \quad \mathcal{S}_{x}-y I>0
$$

The problem (14) is feasible if $y>0$. From (14) a concave function is defined:

$$
q(x):=\sup \left\{y: \mathcal{S}_{x}-y I>0\right\}
$$

Using $q(x)$, problem (15) can be replaced by the equivalent optimization problem:

$$
y_{o p t}=\sup _{x} q(x)
$$

In Ref. 14, a technique has been developed for automatic control problems, which involves a Linear Programming Problem (LPP). The function $q(x)$ is bounded iteratively by a set of hyperplanes, leading to a piecewise linear function $p_{k}(x)$ :

$$
q(x) \leq p_{k}(x):=\min _{1 \leq i \leq k}\left\{a_{i} x-b_{i}\right\}
$$

In the following, it is assumed that there exists a mechanism which checks the constraints and generates the hyperplanes (such a mechanism will be derived in the next part). The algorithm begins with an initial value $y_{l}$ belonging to the feasible set. At iteration $k$ the following LPP is solved:

$$
\max _{x_{\min } \leq x \leq x_{\max }} p_{k}(x)
$$

with $x_{\min }$ and $x_{\max }$ defining some numerical limits of the components of vector $x$. Let $y^{(k)}$ be the solution of this problem. A linear interpolation involving a parameter $\alpha \in[0,1]$ derives a new value of $y$ :

$$
\hat{y}^{(k)}=\alpha y^{(k)}+(1-\alpha) y_{l}
$$

If the set of constraints $\mathcal{S}_{x}-\hat{y}^{(k)} I>0$ is verified (figure $5(\mathrm{a})$ ), the value of $y_{l}$ is replaced by $\hat{y}^{(k)}$ else, new hyperplanes are added (figure $5(\mathrm{~b})$ ), so that a new LPP can be solved at iteration $k+1$. The principle of the CPA is very simple, but the main task is to verify the 
constraints and to generate the hyperplanes.

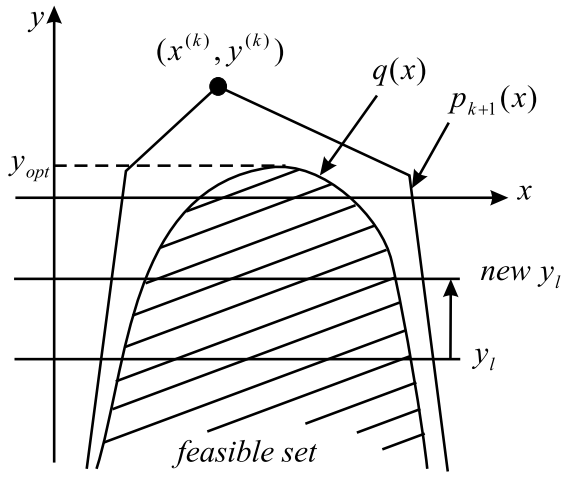

(a)

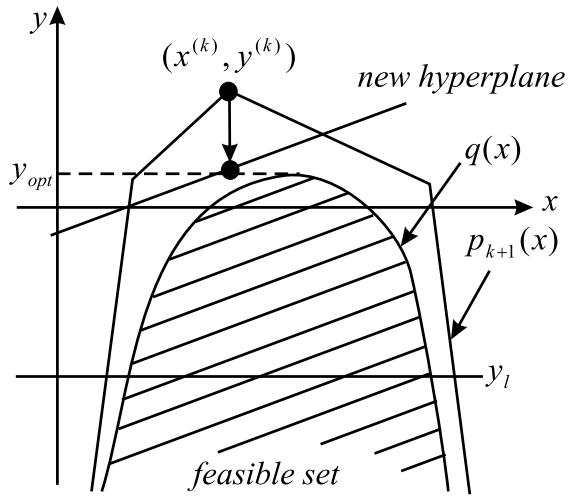

(b)

Figure 5. The CPA in the scalar case

\section{The mechanism for verifying the constraints and generating the hyperplanes}

Since the verification of the constraints and the generation of the hyperplanes are linked, they are considered in the same mechanism. Only general principles are given here: the reader can find in Ref. 13 more information on the different elements involved in the generation of the hyperplanes and their dependance on the criteria specificities.

Two types of constraints have to be considered: in the first case, the constraints are explicit translations of the specifications, so that the verification is done by directly computing the eigenvalues of the corresponding symmetric matrix. A second case arises for frequencydependent constraints, which are translated using some equivalent proposition introduced by the Kalman-Yakubovich-Popov (KYP) lemma: ${ }^{12}$ it allows to replace an infinite number of frequency-dependent constraints by a unique one, using the Hamiltonian matrix $\mathbf{H}$ associated to the constraint. If the Hamiltonian has some eigenvalues on the imaginary axis, they can be reported in the constraint as the frequencies where it is not satisfied.

The generation of the hyperplanes is done using the eigenvectors associated to the negative eigenvalues of the matrix $\mathcal{S}_{x}-\hat{y}^{(k)} I$. For each negative eigenvalue $\lambda_{i}$, a hyperplane is generated from the associated eigenvector $v_{i}$, which verifies:

$$
v_{i}^{T}\left(\mathcal{S}_{x}-\hat{y}^{(k)} I\right) v_{i}<0
$$

Since $\mathcal{S}_{x}$ is affine in $x$, the quadratic product $v_{i}^{T}\left(\mathcal{S}_{x}\right) v_{i}$ has the form:

$$
v_{i}^{T}\left(\mathcal{S}_{x}\right) v_{i}=a_{i}^{T} x+b_{i}
$$


and a hyperplane corresponding to the new added constraint is described by:

$$
a_{i}^{T} x+b_{i}-\left(v_{i}^{T} v_{i}\right) y>0
$$

Section IV gives LMI forms of the different constraints listed in Section II.B, which are suitable for applying the CPA.

\section{Translation of the manufacturer objectives into LMI constraints}

The design specifications contain four temporal constraints, corresponding to the angle of attack, the angle of deflection and its velocity and the consumption; and three frequential ones, where two of them concern the gain and delay margins and the last one the roll-off (i.e. the attenuation of the bending modes). In this part all these constraints are translated into suitable forms to avoid as much as possible any relaxations, which can give the problem unfeasible. Another goal is to preserve the convexity by using LMI formulations which are also suitable to apply the CPA.

Only the expression of the LMI for each constraint is given without specifying the procedure to get them, except for the delay margin: the derivation of the other constraints are presented in Ref. 13.

Remark 3 For the frequency-domain constraints the continuous-time formulation is considered to simplify the application of the KYP lemma. The results are then used by applying first the Tustin transform to the discrete-time closed-loop plant, i.e. by replacing matrices $A_{z w}, B_{z w}, C_{z w}, D_{z w}$ by the following ones:

$$
\begin{aligned}
A_{z w_{c}} & =-\frac{2}{T} I+\frac{4}{T} A_{z w}\left(I+A_{z w}\right)^{-1} \\
B_{z w_{c}} & =\frac{4}{T}\left(I+A_{z w}\right)^{-1} B_{z w} \\
C_{z w_{c}} & =C_{z w}\left(I+A_{z w}\right)^{-1} \\
D_{z w_{c}} & =D_{z w}-C_{z w}\left(I+A_{z w}\right)^{-1} B_{z w}
\end{aligned}
$$

It should be noticed that such a transformation preserves the linearity of the output matrices $C_{z w}$ and $D_{z w}$ with respect to the design variables of the Youla parameter, and does not dispatch them in the other matrices $A_{z w}$ and $B_{z w}$. 


\section{A. Angle of attack}

Consider the wind profiles shown on figure 3. The specification on the angle of attack $i$ has to be considered for each of them, and for each sampling time.

\section{Wind sampling}

To handle the wind velocity $W$ as disturbance input, the discrete-time model of the plant is first completed by considering a first-order hold on input $W$, as suggested in Ref. 21. As it can be seen on figure 6, such an approach gives almost identical frequency responses for the continuous-time plant and the discretized one, which is not the case if a zero-order hold is used.
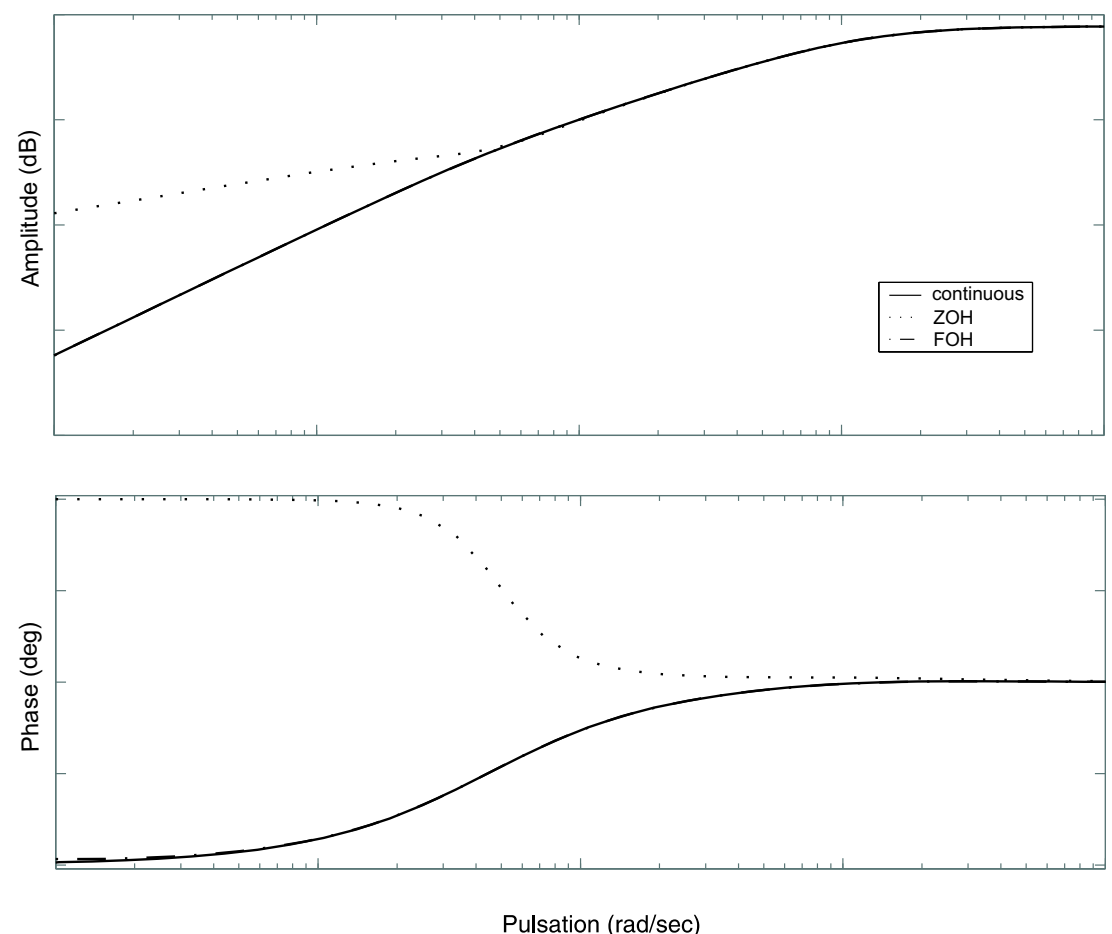

Figure 6. Bode diagrams of the transfer from the wind velocity to the angle of attack.

\section{Application of the $C P A$}

The following objective has to be considered:

$$
i^{2}(n T)<\tau \quad n=0,1, \ldots, 1388
$$

where $T$ is the sample period and $\tau=i_{\max }^{2}$. The number of considered sampling times corresponds to the duration of the atmospheric flight (namely $1388 \times T$ ). 
At each sampling time, this constraint can be written on the following LMI form:

$$
\left(\left(C_{z w}\left(\sum_{k=1}^{n} A_{z w}^{k-1} B_{z w} \hat{w}_{n-k}\right)+D_{z w} \hat{w}_{n}\right)^{*}{ }^{*}\right)^{\prime}>0
$$

where $\hat{w}_{n-k}=\frac{w_{n-k}}{\sqrt{\tau}}, k=0, \ldots n$ and $w_{n}$ are the samples of the wind input.

The constraint (25) should be duplicated 1338 times, in order to respect the specification throughout the atmospheric phase. However, it can more simply be applied only on some critical interval of time, during which the gust of wind acts.

At each iteration of the CPA, the constraint is directly checked by computing the eigenvalues of the matrix (25): the constraint is fullfiled if and only if all eigenvalues are positive. If not, a new hyperplane is generated from the eigenvector associated to the most negative eigenvalue.

\section{B. Consumption}

\section{Formulation as a $\mathcal{H}_{2}$-norm constraint}

The indicator (7) used to represent the consumption strongly depends on the measurement noises which affect the outputs of both sensors, as it can be noticed on figure 7 , where the evolution of $C$ is represented with and without measurement noises.

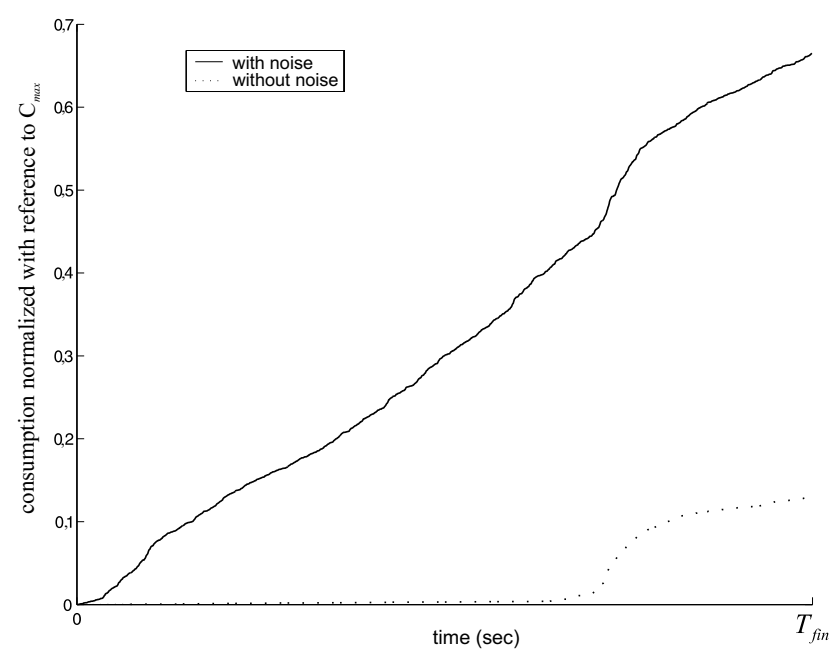

Figure 7. Consumption indicator of the launcher with and without measurement noises.

It is therefore relevant in this section to consider a model of the plant with $\beta_{k}$ as control input and $z_{k}=\beta_{k}-\beta_{k-1}$ as output to be controlled, the measured output $y_{k}$ being corrupted 
by a noise vector $w_{k}$. Such a model writes:

$$
\left\{\begin{aligned}
\left(\begin{array}{c}
x_{k+1} \\
\beta_{k}
\end{array}\right) & =\left(\begin{array}{ll}
A & 0 \\
0 & 0
\end{array}\right)\left(\begin{array}{c}
x_{k} \\
\beta_{k-1}
\end{array}\right)+\left(\begin{array}{c}
B \\
1
\end{array}\right) \beta_{k}=A_{c}\left(\begin{array}{c}
x_{k} \\
\beta_{k-1}
\end{array}\right)+B_{c} \beta_{k} \\
z_{k} & =\left(\begin{array}{cc}
0 & -1
\end{array}\right)\left(\begin{array}{c}
x_{k} \\
\beta_{k-1}
\end{array}\right)+\beta_{k} \\
y_{k} & =\left(\begin{array}{ll}
C & 0
\end{array}\right)\left(\begin{array}{c}
x_{k} \\
x_{k} \\
\beta_{k-1}
\end{array}\right)+\left(\begin{array}{ll}
\nu \\
0 \\
0 & 1
\end{array}\right) w_{k}=C_{c 2}\left(\begin{array}{c}
x_{k} \\
\beta_{k-1}
\end{array}\right)+D_{c} w_{k}
\end{aligned}\right.
$$

where $A, B, C$ are the state space matrices of the discrete-time synthesis model. Parameter $\nu$ is used to weight both noises; it is chosen equal to the asymptotic value of the ratio between the standard deviations $B_{\psi}$ and $B_{\dot{\psi}}$ of the measurement noises on $\psi$ and $\dot{\psi}$, which is equal to 0.219 .

In order to limit the consumption indicator, a constraint on the $\mathcal{H}_{2}$ norm of the transfer between the measurement noises $w_{k}$ and the output $z_{k}$ is now considered.

\section{Application of the $C P A$}

Let $W_{c}$ the controllability gramian of the closed-loop system; its $\mathcal{H}_{2}$ norm is less than $\sqrt{\xi}$ if and only if the following constraint is satisfied:

$$
\left.\frac{1}{\sqrt{\xi}}\left(\begin{array}{cc}
\left(W_{c}^{1 / 2}\right)^{T} & 0 \\
0 & I
\end{array}\right)\left(\begin{array}{c}
C_{z w}^{T} \\
D_{z w}^{T}
\end{array}\right){ }^{*} I\right)^{*}
$$

Note that $W_{c}$ has to be calculated only once time, because it doesn't depend on the design variables. As for the angle of attack, this constraint is simply checked by computing the eigenvalues of the matrix in (27), whereas if the constraint is not satisfied, a new hyperplane is generated from the eigenvector associated to the most negative eigenvalue.

In usual $\mathcal{H}_{2}$ control problems, the value of $\xi$ is minimized under the LMI constraint (27). However, since other constraints have to be considered in the control problem of the launcher, a limitation on the $\mathcal{H}_{2}$ norm will be considered instead of minimising it.

In fact, the following subsection shows that the value of $\xi$ is strongly related to the consumption indicator. 


\section{Analysis and initialization of $\xi$}

It remains to choose the desired maximal value $\xi$ of the $\mathcal{H}_{2}$ norm to be considered. To this end, different controllers have been synthesised by increasing the order of the Youla parameter from an initial controller with poor performance. For each order, the value of $\xi$ is decreased until the problem becomes unfeasible, and the consumption indicator is evaluated for the optimal controller.

Table 2 and Figure 8 show that the relation between the $\mathcal{H}_{2}$ norm $\xi$ and the indicator $C$ is almost affine. It validates the use of the $\mathcal{H}_{2}$ norm to limit the consuption indicator. In the following, an initial value $\xi=0.33$ has been chosen to limit $C$ to (approximately) 0.75 $C_{\max }$ (figure 8).

Table 2. $\mathcal{H}_{2}$-norm $\xi$ and consumption indicator $C$.

\begin{tabular}{|c|c|c|}
\hline$n_{q}$ & $\xi$ & $C / C_{\max }$ \\
\hline 1 & 0,3273 & 0,788 \\
\hline 3 & 0,2908 & 0,698 \\
\hline 5 & 0,2379 & 0,619 \\
\hline 7 & 0,1792 & 0,536 \\
\hline 9 & 0,1290 & 0,463 \\
\hline
\end{tabular}

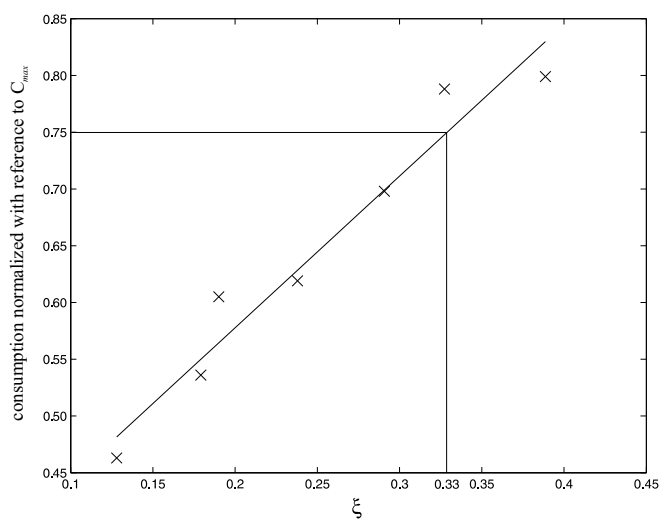

Figure 8. Variation of consumption according to $\xi$.

\section{Roll-off}

This part is the most significant one, because in the preceding syntheses ${ }^{5,4}$ the roll-off constraint was removed for the first flexible mode, which is the most difficult to attenuate. 


\section{Tranformation into a closed-loop constraint on the rigid model}

The problem handled in this subsection is the following: the roll-off specification concerns the open-loop transfer $K G_{s}$ (where $K$ is the controller and $G_{s}$ the flexible model of the launcher), whereas only closed-loop constraints involving the rigid model $G$ can be handled in the proposed approach. Taking into account first that the resonances of the flexible modes are almost equal to $35 \mathrm{~dB}$ in the worst cases configurations, the open-loop constraint on $\left|K G_{s}\right|$ being less than $X_{H F} \mathrm{~dB}$ is replaced by the open-loop contrainst on $|K G|$ being less than $X_{H F}-35 \mathrm{~dB}$; for this small value of gain, the closed-loop transfer $(I-K G)^{-1} K G$ can be considered as well (since $|K G| \ll 1 \Rightarrow(I-K G)^{-1} K G \simeq K G$ ). The considered closed-loop constraint is therefore $\left|(I-K G)^{-1} K G\right|$ being less than $X_{H F}-35 \mathrm{~dB}$.

This transformation, which would be perfect if both $K$ and $G$ were SISO tranfer functions, is not valid in the present case where $G$ is SIMO (with 2 outputs) and $K$ is MISO, since it doesn't take into account any phase consideration: figure 9 shows an example of controlled open-loop response where the previous constraint is satisfied for the rigid model, whereas it is obviously not the case for the flexible one.

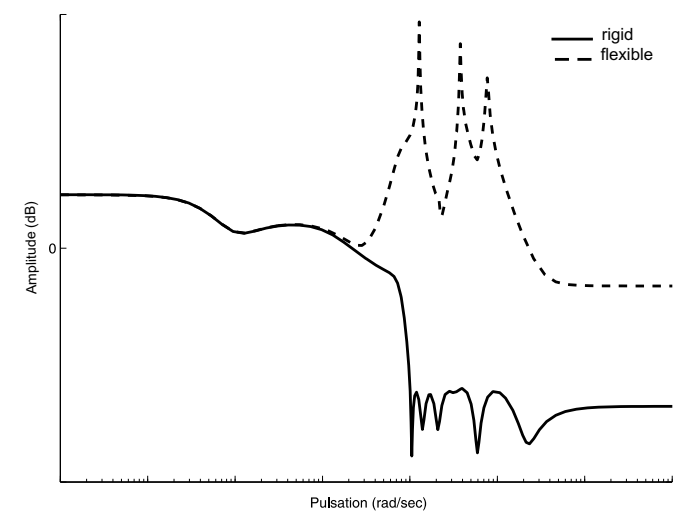

Figure 9. Example of violation of the roll-off constraint.

To solve this problem two solutions have been considered:

- the first solution is to factorize the initial controller into $K=K_{1} K_{0}$ where $K_{0}$ is MISO and $K_{1}$ is SISO and includes the common zeros and poles of both channels (obviously one can choose $K_{0}=K$ and $K_{1}=1$ ). The synthesis is done on the new SISO model $K_{0} G$ (figure 10). In that case, the previous transformation holds and the Youla parameter is also SISO, which is the main interest of this solution. However it introduces a restriction which reduces the space of solutions;

- the second solution consists in considering the attenuation constraint of $X_{H F}-35 \mathrm{~dB}$ on the transfer matrix between the input $\beta$ and the vector of outputs $\left(\begin{array}{ll}\psi & \dot{\psi}\end{array}\right)^{T}$ : the 
same attenuation is then guaranteed for each SISO transfer $\psi / \beta$ and $\dot{\psi} / \beta$. The interest of this solution is to preserve the controller structure. However the Youla parameter having two inputs in that case, the number of state-variables is twice the order chosen for each corresponding transfer.

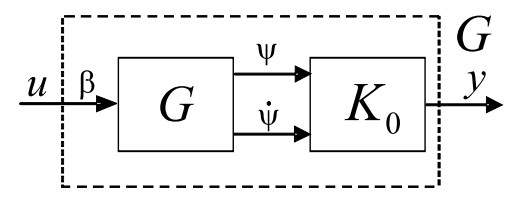

Figure 10. New synthesis model.

Both solutions will be presented in the next section.

\section{Application of the CPA}

According to a slightly modified version of the Kalman-Yakubovich-Popov (KYP) lemma, ${ }^{13}$ the closed-loop constraint $\bar{\sigma}\left(G_{z w}\right)<\gamma$ is satisfied for all $s=j \omega$ in $\left[j \omega_{1}, j \omega_{2}\right]$ if and only if one of the two following equivalent propositions holds:

- $\hat{H}(\omega)>0$ for all $\omega$ in $\left[\omega_{1}, \omega_{2}\right]$, where:

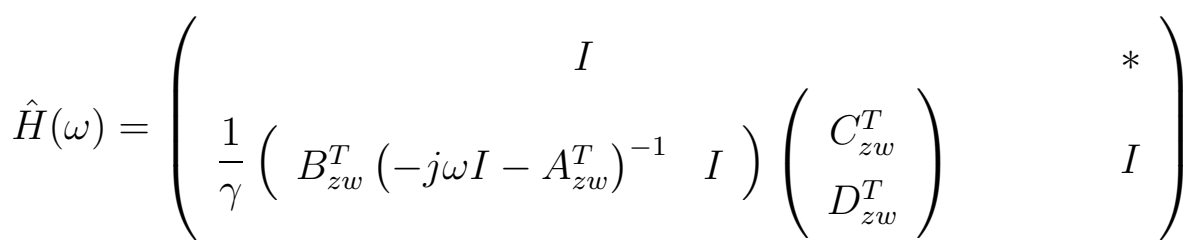

- $R=\gamma^{2} I-D_{z w}^{T} D_{z w}$ is invertible, $\exists \omega_{i} \in\left[\omega_{1}, \omega_{2}\right]$ such that $\hat{H}\left(\omega_{i}\right)>0$, and the Hamiltonian matrix $\mathbf{H}$ has no eigenvalue on the imaginary axis belonging in $\left[j \omega_{1}, j \omega_{2}\right]$, where:

$$
\mathbf{H}=\left(\begin{array}{cc}
A_{z w}+B_{z w} R^{-1} D_{z w}^{T} C_{z w} & B_{z w} R^{-1} B_{z w}^{T} \\
-C_{z w}^{T} C_{z w}-C_{z w}^{T} D_{z w} R^{-1} D_{z w}^{T} C_{z w} & -A_{z w}^{T}-C_{z w}^{T} D_{z w} R^{-1} B_{z w}^{T}
\end{array}\right)
$$

According to these results, verifying that the closed-loop gain is less than $\gamma$ in some frequency domain $\left[\omega_{1}, \omega_{2}\right]$ which includes the natural frequencies of the modes is done by computing the eigenvalues of $\mathbf{H}$. If it has a pure imaginary eigenvalue $j \hat{\omega}$ belonging in $\left[j \omega_{1}, j \omega_{2}\right]$, new hyperplanes are generated by injecting the value $j \hat{\omega}$ in (28) and considering the eigenvectors associated with the negative eigenvalues of $\hat{H}(\hat{\omega})$. 


\section{Stability margins}

\section{General LFT form}

The manufacturer specifications contain three constraints on the stability margins: two of them concern the gain margins and one the delay margin. Using a suitable LFT form ${ }^{13}$ enables to consider that the margin specification is satisfied if and only if the closed-loop plant remains stable for any scalar uncertainty $\delta \in[0,1]$. Thus the Nyquist criterion can be used on the closed-loop plant $G_{z w}$ looped again by $-\delta$ (figure 11).

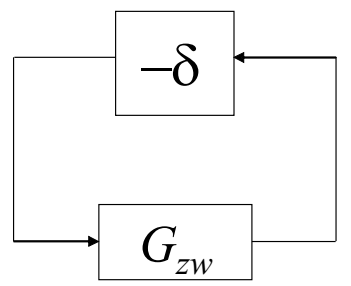

Figure 11. The stability margin formulated as an uncertainty

For the gain margin, such an LFT has been already derived in Refs. 13, 22; the corresponding state space representation of the open-loop plant $G$ to be considered is then:

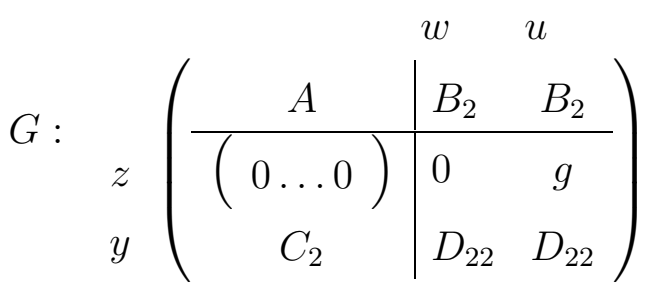

where $g=1-10^{G M / 20}$ specifies the margin to be attained, $G M$ being either equal to $D G M$ or $I G M$ with $\mathrm{dB}$ unit.

It remains to derive a similar LFT form for the delay margin: this is done in the Appendix.

\section{Application of the $C P A$}

From the Nyquist criterion, since $G_{z w}$ is stable, the loop on figure 11 is stable for all $\delta \in[0,1]$ if and only if the Nyquist diagram of $G_{z w}$ does not cut the half line $(-\infty,-1]$ of the real axis. To derive a convex formulation, this constraint is substituted by a harsher one, where the Nyquist diagram must not go into the half-plane to the left of -1 . This later constraint directly becomes a passivity condition if $G_{z w}$ is replaced by $G_{z w}+1$ :

$$
\left(G_{z w}(j \omega)+1\right)+\left(G_{z w}^{*}(j \omega)+1\right) \geq 0 \quad \forall \omega \in[0, \infty)
$$


Using the Kalman-Yakubovich-Popov (KYP) lemma, ${ }^{23}$ this constraint is equivalent to $\hat{H}(\omega)>0$ for all $\omega$ in $[0, \infty)$, where:

$$
\hat{H}(\omega)=\left(\begin{array}{ll}
B_{z w}^{T}\left(-j \omega I-A_{z w}^{T}\right)^{-1} & I
\end{array}\right)\left(\begin{array}{cc}
0 & C_{z w}^{T} \\
C_{z w} & D_{z w}+D_{z w}^{T}+2
\end{array}\right)\left(\begin{array}{c}
\left(j \omega-A_{z w}\right)^{-1} B_{z w} \\
I
\end{array}\right)
$$

It is equivalently satisfied if and only if $R=D_{z w}+D_{z w}^{T}+2>0$ and the Hamiltonian matrix:

$$
\mathbf{H}=\left(\begin{array}{cc}
A_{z w}-B_{z w} R^{-1} C_{z w}^{T} & B_{z w} R^{-1} B_{z w}^{T} \\
-C_{z w}^{T} R^{-1} C_{z w} & -A_{z w}^{T}+C_{z w}^{T} R^{-1} B_{z w}^{T}
\end{array}\right)
$$

has no eigenvalue on the imaginary axis.

As for the roll-off constraint, the stability margin constraint is checked by computing the eigenvalues of $\mathbf{H}$. If it has a pure imaginary eigenvalue $j \hat{\omega}$, new hyperplanes are generated by injecting the value $j \hat{\omega}$ in (32) and considering the eigenvectors associated with the negative eigenvalues of $\hat{H}(\hat{\omega})$.

\section{E. Multiobjective problem}

From all the results exposed above, the multiobjective control problem to be solved is finally the following:

find $x$ under the constraints:

- Angle of attack: (25) for $n=0, \ldots 1388$

- Consumption: (27)

- Roll-off: (28)

- Decreasing gain margin: (32) with open-loop plant (30) and $g=1-10^{D G M / 20}$

- Increasing gain margin: (32) with open-loop plant (30) and $g=1-10^{I G M / 20}$

- Delay margin: (32) with open-loop plant (43)

where vector $x$ contains all the coefficients of the output matrices of the Youla parameter.

Problem (34) is solved for the aerospace launcher in the next section; note that the maximal value of each constraint having been chosen, it is a feasibility problem instead of an optimization problem, where the choice of the criterion could penalize the research of a 
solution.

\section{Application to the launcher control problem}

This section examines the results of the proposed approach on the launcher control problem. The initial controller was synthesized using the loop-shaping $\mathcal{H}_{\infty}$ design approach. ${ }^{24}$ This controller checks the manufacturer specifications, except for the constraint of gain attenuation on the first flexible mode.

The Youla parameter is searched as a Finite Impulse Response (FIR) filter, with an initial order equal to 5. This value is gradually increased as long as problem (34) remains unfeasible, until arriving at a feasible problem or obtaining the numerical unfeasibility.

The numerical interest of the method is pointed out when considering first the first solution of section IV.C (where the plant to be controlled is the open-loop plant in cascade with the initial compensator, see figure 10): indeed it has been able to consider a Youla parameter of order 50 and more without numerical problems, and with an acceptable running time (20 minutes, for the order 50 with an Intel P4 2.53GHz processor and 512 Mo RAM). Merging the Youla parameter with the initial controller gives then a final controller of order 62 .

If now the model in SIMO form is used, with a gain constraint $X_{H F}-35 \mathrm{~dB}$ on each transfer $\psi / \beta$ and $\dot{\psi} / \beta$, the order of the Youla parameter is less significant (22 state variables). The resulting controller is of order 35 .

In order to underline the efficiency of each controller, the main objectives are summarized on figures 12 to 15 , by considering the open-loop frequency responses and the closed-loop time responses to the wind profiles of figure 3, for the nominal values of the parameters and all the worst-case configurations listed in Table 1.

On the frequency responses, the gain margins specifications are summarized by a vertical line above the critical point and the roll-off constraint by an horizontal one; on the time responses, the limitation values on $i, \beta, \dot{\beta}$ and $C$ are explicitely indicated. It is therefore easy to check that all these specifications are satisfied for both controllers. Note finally that the delay margin constraint cannot be checked on the plots but is satisfied in each case, with a value at least equal to 1.2 time the sampling period.

One can note also that the most demanding contraints are the gain margins specifications, the roll-off constraints and the maximal allowable value of the angle of attack; in contrast, the limitations on the deflection, its derivative and the consumption indicator are widely satisfied, although the sensor noises are taken into account. 


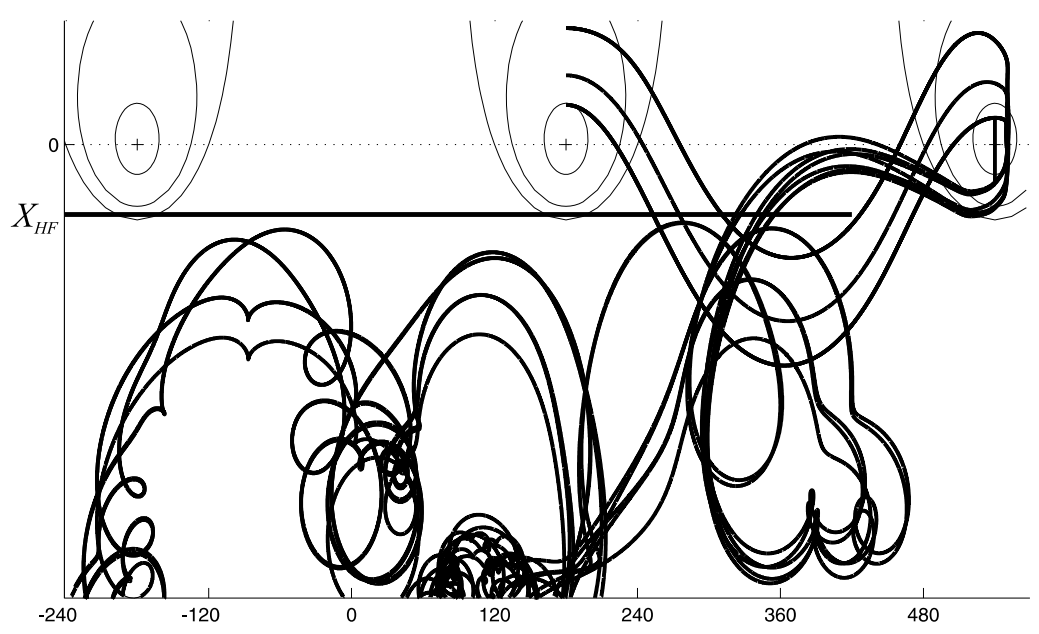

Figure 12. Nichols charts of the worst cases: controller of order 62.

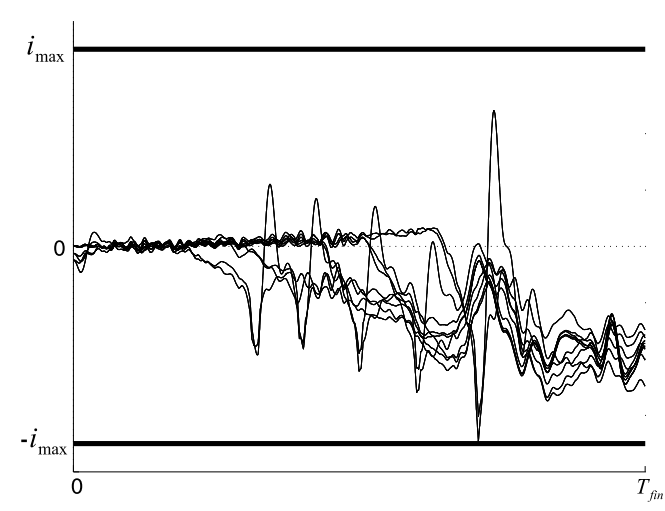

a) Angle of attack

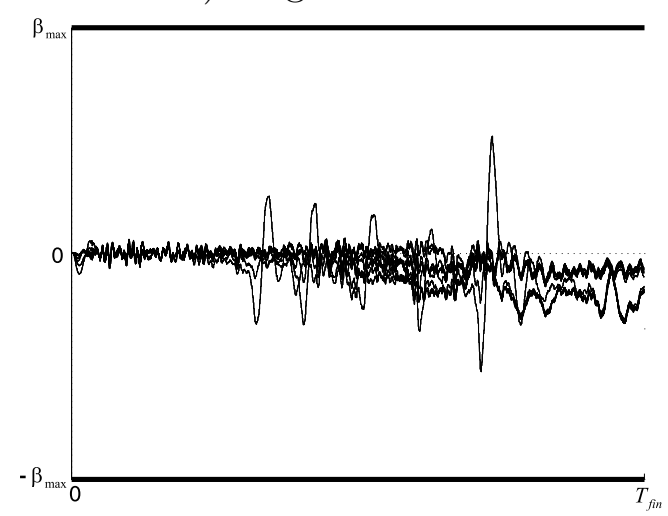

c) Angle of deflection

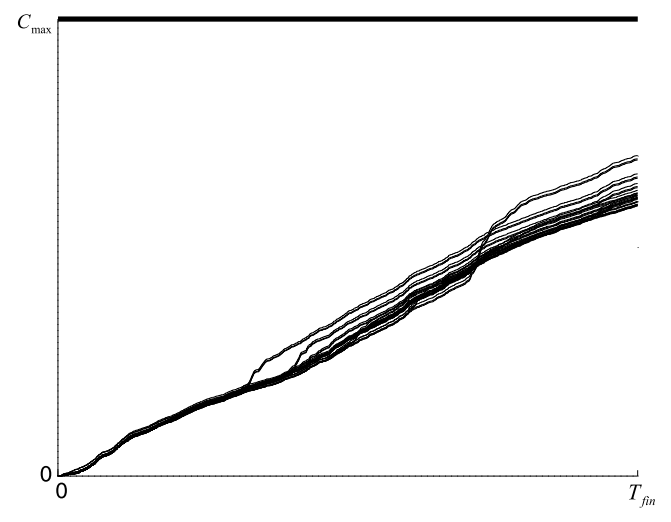

b) Consumption indicator

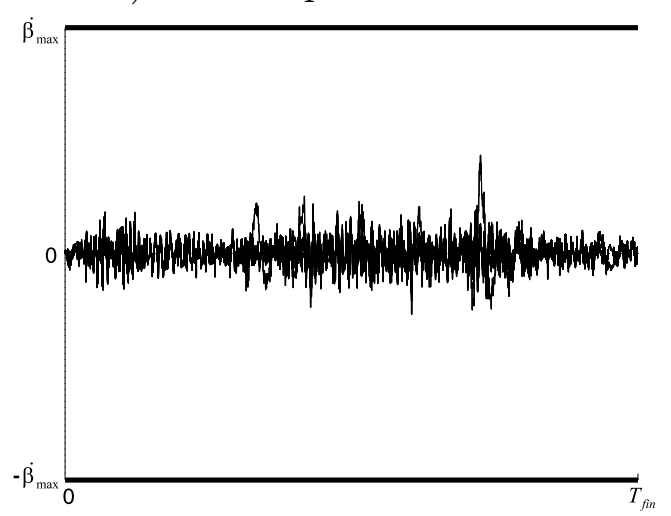

d) Velocity of the angle of deflection

Figure 13. Time responses of the worst cases for all wind gusts: controller of order 62 .

From the Nichols plots, a better attenutation is obtained for the bending modes with the controller of order 35, which gives a higher gap with respect to the required value. This 


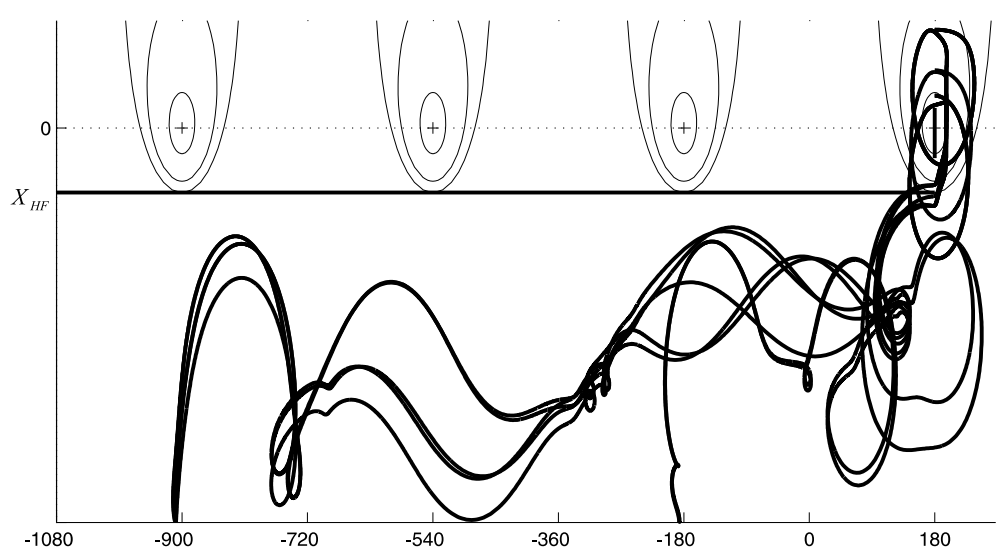

Figure 14. Nichols charts of the worst cases: controller of order 35.

controller also yields a better delay margin, which is at least 1.7 time the sampling period. A similar observation can be made concerning the time responses, where the consumption indicator is less significant; the responses also seem to be less sensitive to the uncertainties.

To see whether the Youla parameters of orders 50 and 22 can be reduced, their impulse responses are presented on figures 16 and 17 (recall that the Youla parameter is an FIR filter). From these figures, it seems difficult to reduce the order of the Youla parameter without changing significantly its impulse response. This observation is checked by decreasing the order gradually with least-squares approximation of the FIR filter with Infinite Impulse Response (IIR) filter. ${ }^{25}$ Applying this procedure confirms the minimality of the order of the Youla parameter, since the reduction of only one state involves several constraints to be not satisfied.

After having checked that a reduction of the impulse response of the Youla parameter is not possible, it can be said that the controller allowing to satisfy all the constraints is of order 62 in the first approach (where a SISO controller is looked for) and 35 in the second. Despite this statement, reducing the order of the controller can be undertaken by methods which should allow simultaneously to take care to the design specifications, which are the subject of forthcoming works. ${ }^{26}$

\section{Conclusion}

In this paper a deep study of a space launcher control problem during the atmospheric stage has been done. The manufacturer specifications are translated to suitable LMI constraints and for each objective physical considerations lead to a good choice of the maximal value of each constraint. Such an analysis is very interesting to answer to the difficult question of the feasibility or unfeasibility of a given set of control specifications. For the same 


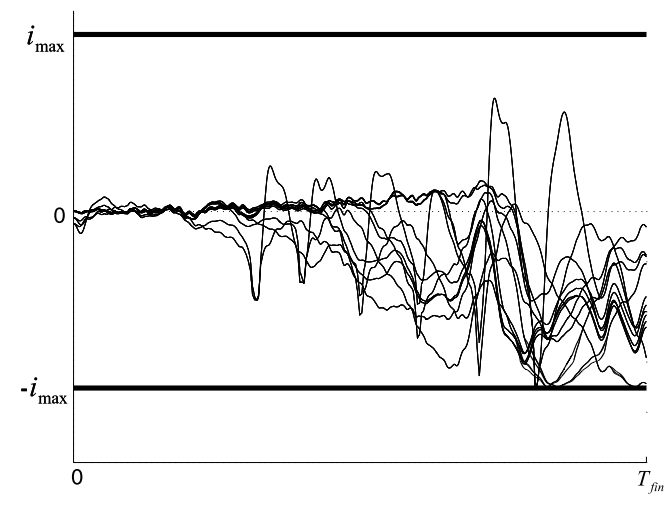

a) Angle of attack

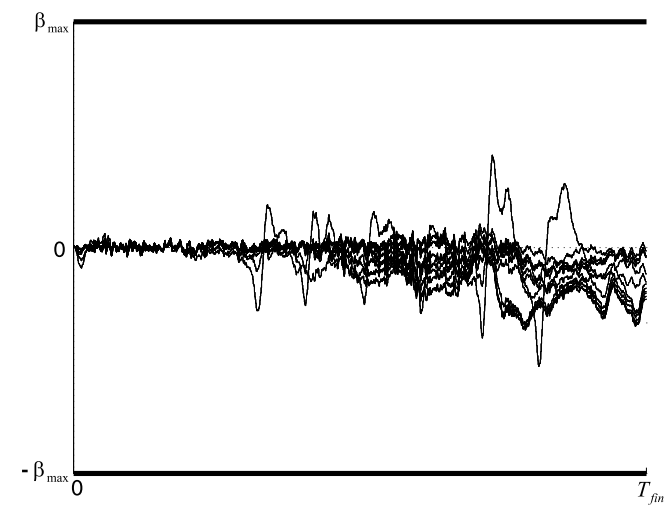

c) Angle of deflection

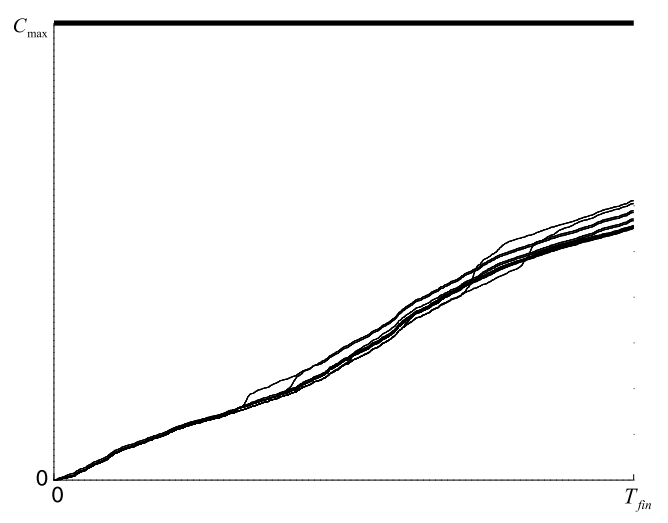

b) Consumption indicator

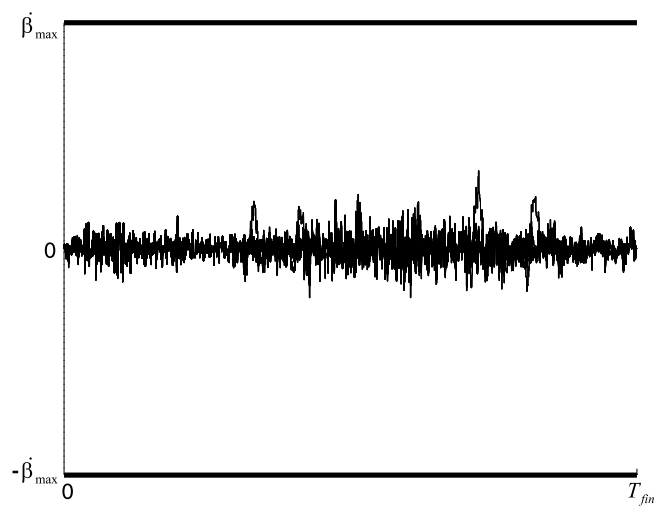

d) Velocity of the angle of deflection

Figure 15. Time responses of the worst cases for all wind gusts: controller of order 35 .

reasons, the development of synthesis tools, where Youla parameter with huge orders can be considered, have been done. Although their development is not the main subject of the paper, their application to the difficult problem considered here has shown the advantages brought by these tools, since a Youla parameter of order 50 was considered without numerical problem (regarding the amount of time calculation and memory space involved). Note also that all specifications are considered using new LMI formulations, which avoid (as much as possible) any relaxation and any introduction of supplementary decision variables.

The methodology developed in this paper considers LTI plants. Since the launcher is time-varying, it will be necessary to perform controller interpolation in order to attain the objectives during the whole atmospheric flight. The development of such a procedure is the subject of forthcoming works. 


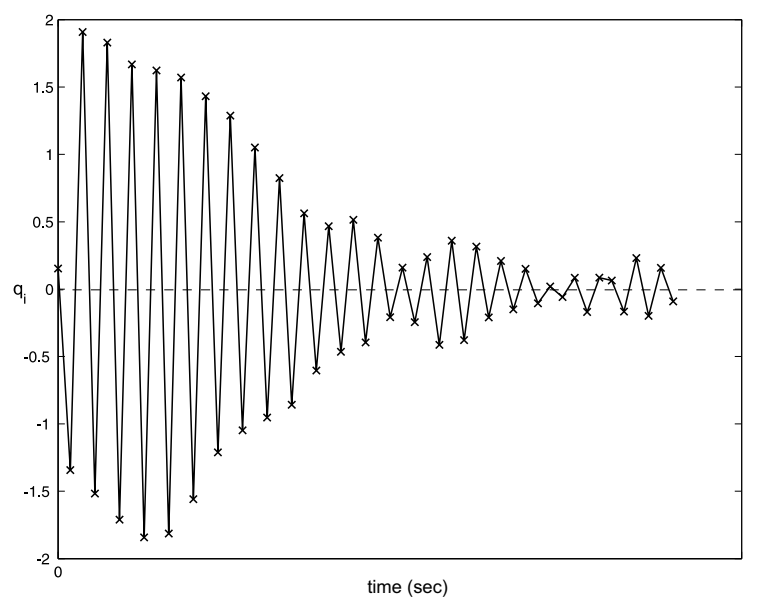

Figure 16. Impulse response of the Youla parameter of order 50.

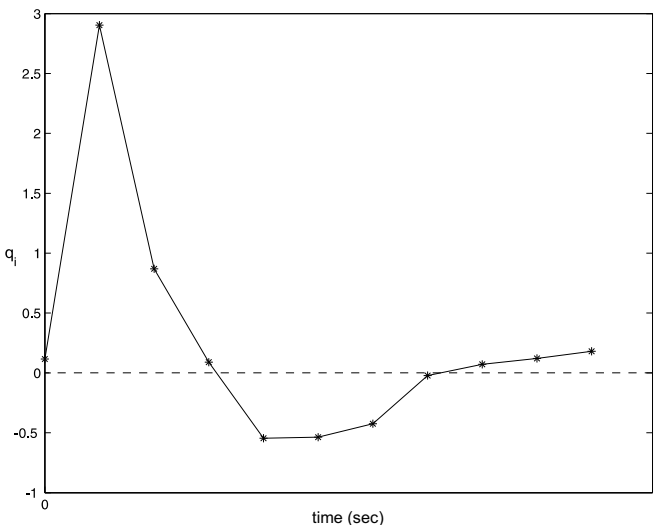

(a) $Q_{1,1}$

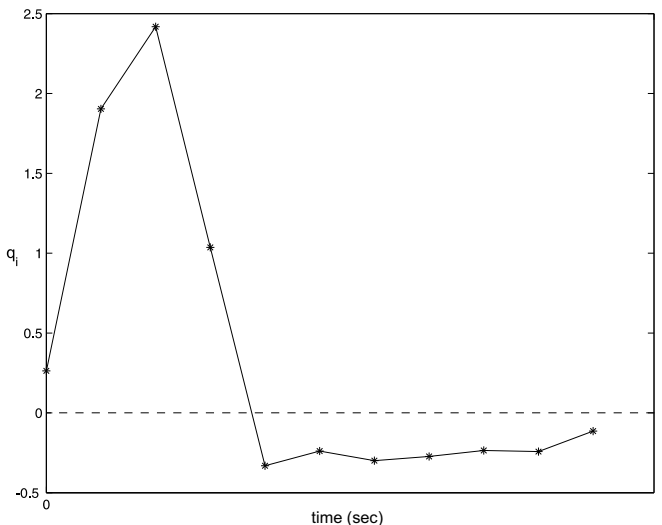

(b) $Q_{1,2}$

Figure 17. Impulse response of the Youla parameter of order 22.

\section{Appendix}

In the manufacturer specifications the delay margin must be at least equal to the sampling period $T$. To handle this constraint the delay must be expressed as an uncertainty $\delta \in[0,1]$ with $\delta=0$ corresponding to the not-delayed system.

Consider a continuous-time system described by the following state-space equation:

$$
\dot{x}(t)=A x(t)+B u(t-\tau) \quad \tau \leq T
$$

with $A \in \mathbb{R}^{n \times n}$ and $B \in \mathbb{R}^{n \times m}$. The corresponding discrete-time model with a zero order hold is obtained by integrating equation (35) between two sampling times:

$$
x_{k+1}=e^{A T} x_{k}+\left(\int_{T-\tau}^{T} e^{A \nu} B d \nu\right) u_{k-1}+\left(\int_{0}^{T-\tau} e^{A \nu} B d \nu\right) u_{k}
$$


Let $B_{1}=\int_{T-\tau}^{T} e^{A \nu} B d \nu$ and $B_{2}=\int_{0}^{T-\tau} e^{A \nu} B d \nu . \quad B_{1}$ and $B_{2}$ can be written in matrix form:

$$
\begin{aligned}
& B_{1}=\left(I_{n} \mid \mathbf{0}_{n m}\right)\left(e^{\hat{A} T}-e^{\hat{A}(T-\tau)}\right)\left(\frac{\mathbf{0}_{n m}}{I_{m}}\right) \\
& B_{2}=\left(I_{n} \mid \mathbf{0}_{n m}\right) e^{\hat{A}(T-\tau)}\left(\frac{\mathbf{0}_{n m}}{I_{m}}\right)
\end{aligned}
$$

with $\hat{A}=\left(\begin{array}{cc}A & B \\ \mathbf{0}_{m n} & \mathbf{0}_{m m}\end{array}\right)$. The representation (36) can be formulated as:

$$
\left(\begin{array}{c}
x_{k+1} \\
u_{k}
\end{array}\right)=\underbrace{\left(\begin{array}{cc}
e^{A T} & B_{1} \\
\mathbf{0}_{m n} & \mathbf{0}_{m m}
\end{array}\right)}_{\tilde{A}}\left(\begin{array}{c}
x_{k} \\
u_{k-1}
\end{array}\right)+\underbrace{\left(\begin{array}{c}
B_{2} \\
I
\end{array}\right)}_{\tilde{B}} u_{k}
$$

$\tilde{A}$ and $\tilde{B}$ are rewritten so that the term $e^{-\hat{A} \tau}$ appears only one time:

$$
\begin{aligned}
& \tilde{A}=\left(\begin{array}{cc|c}
e^{A T} & \left(I_{n} \mid \mathbf{0}_{n m}\right) e^{\hat{A} T}\left(\frac{\mathbf{0}_{n m}}{I_{m}}\right) \\
\mathbf{0}_{m n} & \mathbf{0}_{m m}
\end{array}\right)+ \\
& \left(\begin{array}{c}
-\left(\begin{array}{c}
I_{n} \mid \mathbf{0}_{n m} \\
\mathbf{0}_{m n}
\end{array}\right) e^{\hat{A} T}
\end{array}\right) e^{-\hat{A} \tau}\left(\mathbf{0}_{(n+m) n}\left(\frac{\mathbf{0}_{n m}}{I_{m}}\right)\right) \\
& \left.\tilde{B}=\left(\begin{array}{c}
\mathbf{0}_{n m} \\
I_{m}
\end{array}\right)+\left(\begin{array}{c|c}
I_{n} & \mathbf{0}_{n m} \\
& \mathbf{0}_{m n}
\end{array}\right) e^{\hat{A} T}\right) e^{-\hat{A} \tau}\left(\begin{array}{c}
\mathbf{0}_{n m} \\
I_{m}
\end{array}\right)
\end{aligned}
$$

Defining $\tau=\delta T$, the new variable $\delta$ belongs to $[0,1]$. To be able to put the problem in LFT form, $e^{-\hat{A} \delta T}$ must be linearly approximated, with $\delta=0$ and $\delta=1$ corresponding to matrices $I$ and $e^{-\hat{A} T}$ respectively:

$$
e^{-\hat{A} \delta T} \approx I-\delta\left(I-e^{-\hat{A} T}\right)
$$


Using this expression in (39) gives:

$$
\begin{aligned}
& \tilde{A}=\left(\begin{array}{cc}
e^{A T} & \mathbf{0}_{n m} \\
\mathbf{0}_{m n} & \mathbf{0}_{m m}
\end{array}\right)+\left(\begin{array}{c}
\left(I_{n} \mid \mathbf{0}_{n m}\right)\left(e^{\hat{A} T}-I\right) \\
\mathbf{0}_{m n}
\end{array}\right) \delta\left(\mathbf{0}_{(n+m) n}\left(\frac{\mathbf{0}_{n m}}{I_{m}}\right)\right) \\
& \tilde{B}=\left(\begin{array}{c}
\left(I_{n} \mid \mathbf{0}_{n m}\right) e^{\hat{A} T}\left(\frac{\mathbf{0}_{n m}}{I_{m}}\right) \\
I_{m}
\end{array}\right)+\left(\begin{array}{c}
-\left(I_{n} \mid \mathbf{0}_{n m}\right)\left(e^{\hat{A} T}-I\right) \\
\mathbf{0}_{m n}
\end{array}\right) \delta\left(\begin{array}{c}
\mathbf{0}_{n m} \\
I_{m}
\end{array}\right)
\end{aligned}
$$

Taking into account the fact that $\delta$ is a scalar and considering the following matrices:

$$
\left.\begin{array}{l}
\tilde{A}_{0}=\left(\begin{array}{cc}
e^{A T} & \mathbf{0}_{n m} \\
\mathbf{0}_{m n} & \mathbf{0}_{m m}
\end{array}\right) \\
\tilde{B}_{0}=\left(\begin{array}{c|c}
\left(I_{n} \mid \mathbf{0}_{n m}\right.
\end{array}\right) e^{\hat{A} T}\left(\frac{\mathbf{0}_{n m}}{I_{m}}\right) \\
I_{m}
\end{array}\right)\left(\begin{array}{c}
-\left(I_{n} \mid \mathbf{0}_{n m}\right)\left(e^{\hat{A} T}-I\right) \\
\mathbf{0}_{m n}
\end{array}\right)\left(\begin{array}{c}
\mathbf{0}_{n m} \\
I_{m}
\end{array}\right)
$$

the state-space equation (38) can be written:

$$
\left(\begin{array}{c}
x_{k+1} \\
u_{k}
\end{array}\right)=\tilde{A}_{0}\left(\begin{array}{c}
x_{k} \\
u_{k-1}
\end{array}\right)+\tilde{B}_{0} u_{k}+L(-\delta)\left(u_{k-1}-u_{k}\right)
$$

which corresponds to the LFT form of figure 18. The open-loop state-space representation to be considered is therefore:

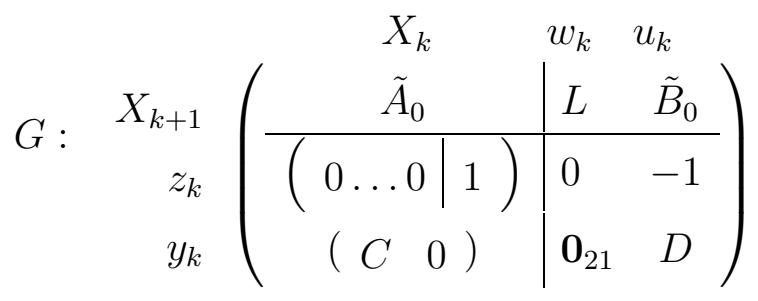




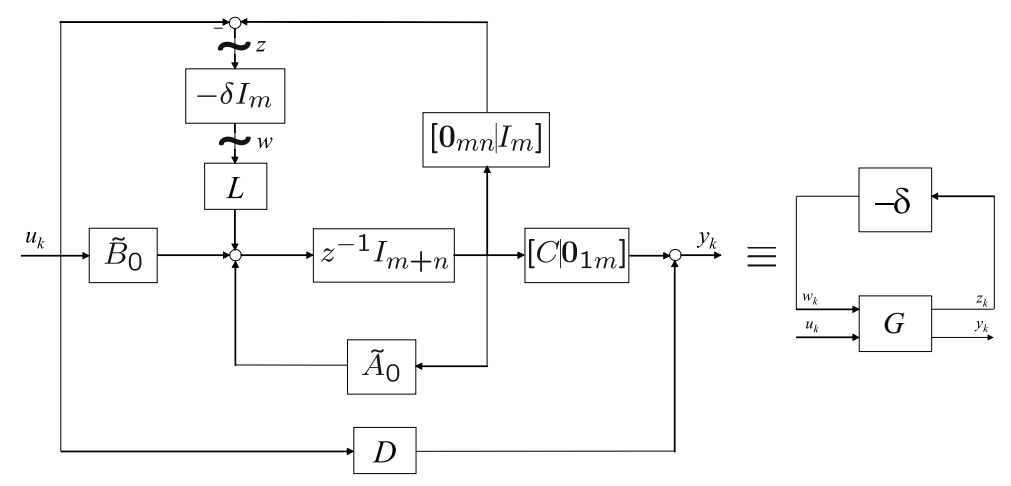

Figure 18. LFT form related to the delay margin.

\section{References}

${ }^{1}$ Mauffrey, S., Chabert, F., and Schoeller, M., " $\mathrm{H}_{\infty}$ method applied to launcher control during atmospheric flight," 3rd Intern. Conf. on Spacecraft Guidance, Navigation and Control Systems, ESTEC, Noordwijk, The Netherlands, november 1996, pp. 349-354.

${ }^{2}$ Mauffrey, S. and Schoeller, M., "Non-Stationary $\mathrm{H}_{\infty}$ control law for launcher with bending modes," 14th IFAC Symp. on Automatic Control in Aerospace, Seoul, Korea, 1998, pp. 433-438.

${ }^{3}$ Olivia, A. P. and Leite Filho, W. C., "Eigenstructure versus optimal control for decoupling," Control Engineering Practice, Vol. 10, No. 10, 2001, pp. 1059-1079.

${ }^{4}$ Voinot, O., Alazard, D., Apkarian, P., Mauffrey, S., and Clement, B., "Launcher Attitude Control: Discrete-time Robust Design and Gain-scheduling," Control Engineering Practice, Vol. 11, No. 11, November 2003, pp. 1243-1252.

${ }^{5}$ Clement, B., Duc, G., and Mauffrey, S., "Aerospace Launch Vehicle Control: a Gain-scheduling Approach," Control Engineering Practice, Vol. 13, No. 3, March 2005, pp. 333-347.

${ }^{6}$ Arzelier, D., Clement, B., and Peaucelle, D., "Multiobjective $\mathcal{H}_{2} / \mathcal{H}_{\infty} /$ Impulse-to-Peak Control of a Space Launch Vehicle," European Journal of Control, Special Issue on Linear Matrix Inequalities in Control, Vol. 12, No. 1, 2006, pp. 57-70.

${ }^{7}$ Boyd, S. and Barratt, C., Linear Controller Design - Limits of Performance, prentice-hall, upper saddle river, nj ed., 1991.

${ }^{8}$ Scherer, C., Gahinet, P., and Chilali, M., "Multiobjective output-feedback control via LMI optimization," IEEE Trans. Automat. Contr., Vol. 42, No. 7, 1997, pp. 896-911.

${ }^{9}$ Lee, J. H., "Nonlinear programming approch to biaffine matrix inequality problems in multiobjective and structured control," IEEE American Control Conference, San Diego, California, June 1999, pp. 18171821.

${ }^{10}$ Oliveira, M. C., Geromel, J. C., and Bernussou, J., "Extended $\mathrm{H}_{2}$ and $\mathrm{H}_{\infty}$ characterizations and controller parametrizations for discrete-time systems," International Journal of Control, Vol. 75, No. 9, June 2002, pp. 666-679.

${ }^{11}$ Maciejowski, J. M., Multivariable feedback design, addison-wesley, wokingham ed., 1990.

${ }^{12}$ Boyd, S., Ghaoui, L. E., Feron, E., and Balakrishnan, V., Linear matrix inequalities in system and control theory, siam, philadelphia ed., 1994. 
${ }^{13}$ Abbas-Turki, M., Duc, G., and Clement, B., "Multiobjective synthesis using LMI formulations for application of the cutting plane algorithm," European Journal of Control, Special Issue on Linear Matrix Inequalities in Control, Vol. 12, No. 1, 2006, pp. 40-56.

${ }^{14}$ Kao, C.-Y., Efficient Computational Methods for Robustness Analysis, Ph.D. thesis, Massachusetts Institute of Technology, September 2002.

${ }^{15}$ Clement, B., Duc, G., Mauffrey, S., and Biard, A., "Gain scheduling for an aerospace launcher with bending modes," 15 IFAC Symposium on Automatic Control in Aerospace, Bologna, September 2001, pp. $475-480$.

${ }^{16}$ Abbas-Turki, M., Duc, G., and Clement, B., "Robust control of a space launcher by introducing LQG/LTR ideas in the NCF robust stabilisation problem," $16^{\text {th }}$ IFAC Symposium on Automatic Control in Aerospace, St-Petersburg, June 2004.

${ }^{17}$ Raggazini, J. R. and Franklin, G. F., Sampled-Data control systems, macgraw-hill, new-york ed., 1958.

${ }^{18}$ Hindi, H. A., Hassibi, B., and Boyd, S. P., "Multiobjective $\mathcal{H}_{2} / \mathcal{H}_{\infty}$-optimal control via finite dimensional Q-Parametrization and linear matrix inequality," IEEE American Control Conference, Philadelphia, Pensylvania, June 1998, pp. 3244-3249.

${ }^{19}$ Scherer, C. W., "From Mixed to Multi-Objective Control," $38^{\text {th }}$ IEEE Conference on Decision 83 Control, Phoenix, Arizona, 1999, pp. 3621-3626.

${ }^{20}$ Alazard, D. and Apkarian, P., "Exact observer-based structures for arbitrary compensation," Int. J. Robust Nonlinear Control, Vol. 9, No. 9, 1999, pp. 101-118.

${ }^{21}$ Imbert, N., "Robustness analysis of a launcher attitude controller via $\mu$-analysis," 15th IFAC Symposium on Automatic Control in Aerospace, Bologna, 2001, pp. 481-486.

${ }^{22}$ Abbas-Turki, M., Duc, G., and Clement, B., "LMI formulation for the feasibility decision on the time template and stability margins consideration," Joint IEEE Conf. on Decision and Control and European Control Conf., Sevilla, December 2005.

${ }^{23}$ Ionescu, V. and Stocia, A., Robust stabilization and $H^{\infty}$ problems, kluwer academic publishers, boston ed., 1999.

${ }^{24}$ Abbas-Turki, M., Etude de Faisabilité d'un Cahier des Charges en Automatique : Application au Pilotage d'un Lanceur Spatial, Ph.D. thesis, Université Paris XI Orsay, 2005.

${ }^{25}$ H.Brandenstein and Unbehauen, R., "Least-square approximation of FIR by IIR filters," IEEE Trans. Signal Processing, Vol. 46, No. 1, 1998, pp. 21-30.

${ }^{26}$ Abbas-Turki, M., Duc, G., and Clement, B., "Retouche de correcteur multiobjectifs par optimisation convexe," to appear, Journal Européen des Systèmes Automatisés, 2006. 